\title{
Keynote Lectures
}

[KN11] X-ray FEL instrumentation for structure and dynamics of biomolecules $\underline{\text { Sébastien Boutet }}$

Linac Coherent Light Source, SLAC National Accelerator Laboratory

Since 2009, the Linac Coherent Light Source has been producing ultrashort and ultra-intense pulses of x-rays in the 2 to $0.1 \mathrm{~nm}$ wavelength range, thus becoming the first operational X-ray Free Electron Laser. It has had immediate impact in the world of structural biology in the fields of crystallography and imaging. With the uniqueness of the XFEL sources, there comes an inevitable need for new unique instrumentation capable of fully utilizing these capabilities. In this presentation, XFEL instrumentation for structural biology studies will be presented along with some recent results utilizing this instrumentation. 